\title{
Aripiprazole en el tratamiento sintomático del síndrome de Gilles de la Tourette (enfermedad de los tics)
}

\author{
Marcelo Miranda $\mathrm{C}^{1}$, Claudia Castiglioni $\mathrm{T}^{2}$. \\ Aripiprazole for the treatment \\ of Tourette syndrome. \\ Experience in 10 patients
}

Background: Tourette syndrome is a neuropsychiatric disorder characterized by motor and vocal tics, attentional deficit, poor control of impulses and obsessive compulsive disorder. Pharmacological treatment is often disappointing due to partial response and frequent poor tolerance to neuroleptic drugs which are otherwise the most effective therapy so far. Aim: To report a lasting improvement obtained with a new drug, aripiprazole that acts modulating both dopaminergic and serotoninergic neurotransmission. Material and methods: Ten patients refractory to their usual therapy, aged 10 to 35 years, were switched to aripiprazole in an open trial. Results: Nine of the 10 patients showed a significant response assessed by the Yale severity tics rating scale and the clinical global impression scale $(p<0.01)$. No relevant adverse effects were observed. Conclusions: Aripiprazole may be a good pharmacological option for patients with Tourette syndrome (Rev Méd Chile 2007; 135: 773-6).

(Key words: Aripiprazole; Neurotransmitter agents; Tourette syndrome)

\begin{abstract}
Recibido el 24 de octubre, 2006. Aceptado el 14 de diciembre, 2006.
${ }^{1}$ Unidad de Neurología, Clínica Las Condes. Fundación Parkinson y Alzheimer. ${ }^{2}$ Departamento de Pediatría. Unidad de Neurología Infantil, Clínica Las Condes.
\end{abstract}

Conflictos de Interés: Los autores declaran no tener conflictos de interés con la industria que comencializa el fármaco en el país.

Los

os tics pueden ser definidos como fragmentos desinhibidos y aislados de actos motores normales, que se traducen por actividad motora (tics motores) o por presencia de vocalizaciones (tics vocales) ${ }^{1}$. Se caractenzan por la posibilidad de inhibirlos voluntariamente por un lapso breve y ser precedidos frecuentemente por una imperiosa necesidad de realizarlos. Muy frecuentes de observar en la pobla-

Correspondencia a: Marcelo Miranda C. Lo Fontecilla 441. E-mail.marcelomirandac@gmail.com ción general, se estima que $21 \%$ a $24 \%$ de los niños presenta tics en algún momento (lo que se conoce como tics transitorios de la infancia) ${ }^{2}$, y entre $2 \%$ y $5 \%$ los mantienen en forma crónica ${ }^{1}$. Cuando estos tics motores se prolongan por más de un año y se asocian con tics vocales, el cuadro recibe el nombre de síndrome de Gilles de la Tourette ${ }^{1}$, en honor al médico francés que describió el cuadro en 1885. No es tan infrecuente que persistan en la edad adulta y en este grupo etario pueden ser particularmente invalidantes y afectar la calidad de vida. El único estudio existente en nuestro medio demostró una casuística 
importante en pocos meses de enrolamiento, lo que apoya que esta condición es prevalente ${ }^{3}$.

Por largo tiempo, este trastomo fue considerado de origen psicógeno o reflejo de un trauma sexual, según psiquiatras como Freud y Mahler ${ }^{5}$. Sólo en la década 1960-69 comienza la interpretación biológica de este síndrome. La base fisiopatológica del síndrome se desconoce aún, pero la hipótesis más aceptada es la que plantea una hiperactividad de la neurotransmisión dopaminérgica asociada a una sobreactivación límbica del sistema motor ${ }^{4}$. Esto explica la utilidad de fármacos que antagonizan esta actividad como los neurolépticos. Sin embargo, cuando se requiere un tratamiento farmacológico sintomático de los tics en el síndrome de Tourette, la respuesta frecuentemente es parcial o transitonia y los pacientes a menudo no toleran los efectos adversos que le provocan estos fármacos, especialmente en el ánimo y función cognitiva. Prácticamente cada nuevo neuroléptico es usado en el tratamiento de esta enfermedad. La refractariedad de algunos enfermos ha llevado incluso al uso de cirugía con estimulación cerebral profunda para modular la actividad neuronal anormal de los ganglios basales y tálamo.

Aripiprazole es un nuevo neuroléptico con un mecanismo distinto de acción, ya que no es antagonista de los receptores dopaminérgicos tipo 2 , sino agonista parcial de estos receptores $\mathrm{y}$, a la vez, agonista de receptores serotoninérgicos 5HT (5hidroxitriptamina) tipo $1^{\underline{a}}$ y bloqueador de receptores $5 \mathrm{HT}^{6}{ }^{6}$. Esta acción moduladora dual, tanto de la actividad serotoninérgica como dopaminérgica, explicaría su utilidad en el control de síntomas ansiosos depresivos y el tener un perfil menor de efectos adversos. En la literatura existe una escasa experiencia, pero favorable con este fármaco como terapia del síndrome de Tourette y sus comorbilidades neuropsiquiátricas ${ }^{7-9}$. Recientemente, se publicó en el Reino Unido una experiencia en que describe la utilidad del fármaco en 11 pacientes refractarios a la terapia habitual ${ }^{7}$. El objetivo de esta comunicación es describir la respuesta motora en una serie de 10 pacientes con síndrome de Gilles de la Tourette refractario a otras terapias.

\section{PACIENTES y MÉTodo}

El estudio fue prospectivo, sin placebo y no ciego. Se consideraron pacientes controlados en forma ambulatoria con el síndrome de Tourette durante un periodo de enrolamiento de 3 meses desde enero de 2006. Todos los pacientes cumplían los criterios diagnósticos del síndrome, de acuerdo al grupo internacional de estudio del síndrome de Tourette $^{10}$. Estos criterios incluyen la presencia de tics motores y vocales, persistentes por más de 1 año, no necesariamente contemporáneos, de inicio antes de los 21 años y sin una etiología definida. Se empleó el fármaco en 10 pacientes con una forma severa del síndrome, rango de edad 10-35 años, todos presentaban un síndrome de Tourette refractario al uso de fármacos como haloperidol, pimozide, sulpiride, olanzapina, tetrabenazina, levetiracetam, pergolide, toxina botulínica e incluso una fallida cirugía estereotáxica bilateral.

Previo consentimiento informado, y con aprobación del Comité de Ética Clínica Las Condes, los pacientes recibieron aripiprazole en dosis de 5 a 25 $\mathrm{mg} /$ día, la que se adicionó a la terapia habitual del paciente, esta última fue reducida gradualmente en 1 a 2 meses. La respuesta se evaluó al cabo de 1 mes de tratamiento con la escala de tics de Yale y la escala de impresión clínica global. La escala de tics de la Universidad de Yale evalúa en 10 ítems aspectos como la severidad, frecuencia, impacto en la vida diaria y complejidad de los tics ${ }^{1}$. Su valor máximo es 60 puntos. La escala de impresión clínica objetiva la severidad de cualquier enfermedad neuropsiquiátrica desde 1 (normal) a 6 (pacientes más graves) y el cambio con la intervención desde 1 (muy mejorado) hasta 7 (empeoramiento severo) ${ }^{10}$.

Se aplicó la t de Student para determinar la significación estadística de los cambios observados.

\section{RESUltados}

Nueve de 10 pacientes mostraron una respuesta significativa $(p<0,001)$ en las escalas evaluadas (Figuras 1 y 2). Sólo un paciente, el que recibió una fallida cirugía, tuvo una respuesta insatisfactoria. La dosis promedio efectiva alcanzó los $10 \mathrm{mg} /$ día (rango 7,5$25 \mathrm{mg} /$ día). En la mitad de los pacientes pudo observarse una respuesta favorable ya a la semana de terapia. Un paciente presentó efectos extrapiramidales de tipo parkinsoniano, y correspondió al paciente de mayor edad. Estas manifestaciones extrapiramidales se revirtieron con la reducción de la dosis. Este paciente adulto logró reintegrarse a su 
trabajo luego de 2 años de estar severamente afectado por tics. Otro paciente presentó náuseas y vómitos en forma transitoria al iniciar el tratamiento, estas molestias cedieron con reducción de la dosis del fármaco. No ha habido aumento de peso en los enfermos. El seguimiento ha alcanzado 8 meses.

\section{DisCUSIÓN}

Esta experiencia clínica sugiere que aripiprazole puede ser una alternativa a considerar en el tratamiento sintomático del síndrome de Tourette. Este estudio presenta varias limitaciones, al no ser controlado con placebo ni ciego. Se enfocó fundamentalmente a evaluar el aspecto motor del síndrome, ya que éste fue el motivo de consulta

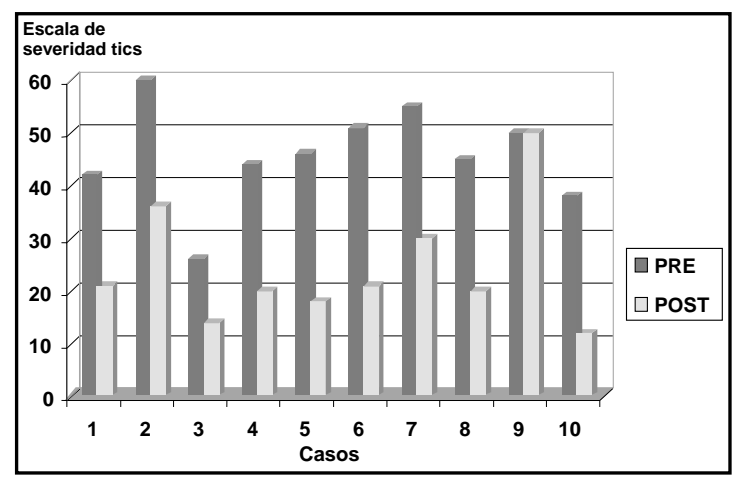

Figura 1. Muestra los cambios observados en la escala de severidad de tics luego de 1 mes de terapia. Nueve de 10 pacientes mostraron cambios significativos ( $p<0,01$ ).

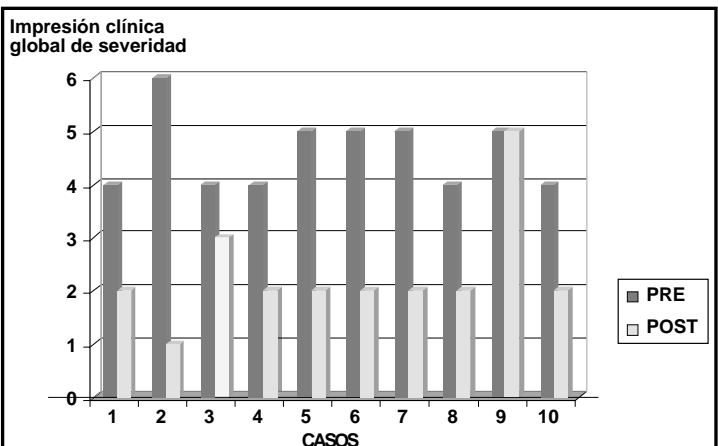

Figura 2. Muestra los cambios observados en la escala de impresión clínica de severidad luego de 1 mes de tratamiento. Nueve de 10 pacientes mostraron cambios significativos ( $p \varangle 0,01$ ). de los pacientes y no se objetivó la comorbilidad psiquiátrica. La serie de pacientes es reducida porque seleccionamos pacientes con una forma severa del síndrome, a pesar de esto, nuestro estudio es el segundo con mayor número de enfermos comunicado en la literatura. Nuestro trabajo junto al de Davies y cols ${ }^{7}$, son de los pocos ensayos en que se estudia objetivamente la respuesta al tratamiento con los cambios en escalas validadas internacionalmente. Aun considerando la característica variabilidad y remisión espontánea que pueden tener los tics en estos pacientes, este grupo de enfermos en particular, se caracterizó por la severidad y mantención en el tiempo de tics muy graves, en los que la mejoría posterior a la intervención con aripiprazole se ha mantenido por un periodo importante e inusual en la historia natural del trastorno para cada paciente. El perfil de seguridad del fármaco debe controlarse con más experiencia y tiempo de uso, ya que éste no estaría exento de generar diskinesia tardía o el síndrome neuroléptico maligno ${ }^{11}$. A diferencia de otros antipsicóticos atípicos, aripiprazole no prolongaría el intervalo QT, no produce hiperprolactinemia ni cambios en los niveles de lípidos o glucosa sanguínea, o en el peso corporal, lo que le otorga una ventaja en su perfil de efectos adversos 6 . Estos parámetros específicos, salvo el peso corporal, no fueron evaluados en el presente estudio.

La respuesta parcial que se obtiene con los diversos fármacos en el manejo de este síndrome, explica el nacimiento de otras altemativas de tratamiento propuestas en los últimos años con resultado dispar. La terapia conductual que pretende lograr que el paciente tome conciencia de sus tics y de las sensaciones previas a su aparición y que intente realizar movimientos antagónicos o que inhiban los tics aparece como promisoria ${ }^{1}$. Por otro lado, se ha intentado con estimulación magnética transcraneal modificar la actividad neuronal intracortical de tipo inhibitoria que estanía defectuosa en estos pacientes, los resultados no han sido satisfactorios ${ }^{13}$.

Aripiprazole es un nuevo antipsicótico, que fue aprobado por la Food and Drug Administration de los Estados Unidos de Norteamérica en el año 2002 para el tratamiento de la esquizofrenia. El mecanismo de acción aceptado para los antipsicóticos típicos, como haloperidol, es el bloqueo de los receptores dopaminérgicos tipo 2; para los antipsicóticos atípicos 
el bloqueo serotoninérgico y dopaminérgico $\mathrm{y}$, últimamente, en relación con los llamados antipsicóticos de tercera generación, como el anipiprazole, su mecanismo de acción novedoso se debe a que actúan como agonistas parciales de receptores D2 por lo que también se les ha denominado sestabilizadores del sistema dopaminérgico $\emptyset$. Un agonista parcial es una droga que actuando sobre el receptor puede antagonizarlo o puede activarlo, es decir que puede actuar como antagonista o como agonista del receptor, dependiendo de la cantidad de dopamina disponible en el momento. Si hay excesiva dopamina el agonista parcial actúa como antagonista del receptor (bloqueándolo), si hay escasa dopamina el agonista parcial actúa como agonista del receptor (activándolo) ${ }^{6}$. Por su mecanismo de acción el aripiprazole disminuye la actividad dopaminérgica excesiva en la vía mesolímbica, mejorando los síntomas positivos de las psicosis y aumenta la actividad dopaminérgica deficiente en la vía mesocortical dinigidas a regiones prefrontales, mejorando los síntomas cognitivos y afectivos de la esquizofrenia ${ }^{6}$.

Existe acuerdo que a nivel cerebral se distinguen varios circuitos neuronales paralelos que dirigen

\section{REFERENCIAS}

1. Leckman J, King R, Cohen D. Tics and Tic disorders en Leckman J, Cohen D Eds. Tourette Syndrome: Tics, Obsessions, Compulsions. New York. Wiley \& Sons 1999; 23-42.

2. Pringsheima T, Jeptha W, Lang A. Tics. Curr Opin Neurol 2003; 16: 523-7.

3. Miranda M, MenÉndez P, David P, Troncoso M, Hernández M, Chana P. Enfermedad de los Tics. Rev Méd Chile 1999; 127: 1480-6.

4. BohlHALter S, GoldFine M, Matteson S. Neural comelates of tic generation in Tourette syndrome: an eventrelated functional MRI study. Brain 2006; 129: 2029-37.

5. KUSHNER H. A Cursing Brain: The Histories of Tourette Syndrome. London. Harvard University Press. 1999: 10-193.

6. LEBERMAN J. Anipiprazole en Schatzberg A, Nemeroff Ch. Eds. Textbook of Psychopharmacology, Washington, American Psychiatric Publishing 2004: 487-94.

7. Davies L, Stern JS, Agrawal N, Robertson MM. A case series of patients with Tourette's syndrome in the United Kingdom treated with Aripiprazole. Hum información desde la corteza a estructuras subcorticales (ganglios basales) y de regreso a la corteza pasando por el tálamo (circuitos corteza-ganglios basales-tálamo-corteza) ${ }^{14}$. Estos circuitos median tanto la actividad motora, sensonial, emocional como la cognitiva. Se ha planteado que los pacientes con síndrome de Tourette tendrían una desinhibición de estos circuitos que a nivel motor se expresańa como tics y compulsiones y a nivel del circuito límbico y frontal como parte de la sintomatología obsesiva, trastorno atencional, descontrol de impulsos entre otras psicomorbilidades ${ }^{15}$. Es posible plantear que el efecto favorable de aripiprazole se explique finalmente por una modulación de la actividad de estos circuitos disfuncionales en el síndrome de Tourette cuya actividad es mediada en forma importante por dopamina y serotonina.

En conclusión, nuestra experiencia favorece la utilidad de aripiprazole en el control de la sintomatología motora del síndrome de Tourette.

Esta experiencia debe ser validada con estudios controlados con placebo como los que están ya en ejecución por la Asociación de Tourette Americana.

Psychopharmacol 2006; 21: 447-53.

8. Bubl E, Perlov E, Tebartz Van Eust. Aripiprazole in patients with Tourette syndrome. World J Biol Psychiatry 2006; 7: 123-5.

9. DuANE DD. Aripiprazole in childhood and adolescence for Tourette syndrome. J Child Neurol 2006; 21: 358.

10. The Tourette Syndrome Ciassification Study Group. Arch Neurol 1993; 50: 1013-16.

11. Guy W. Assessment Manual for Psychopharmacology - Revised Rockville, MD, 1976, pp 218-222.

12. Maytal G, Ostacher G, Stern TA. Anipiprazole-related tardive dyskinesia. CNS Spectr 2006; 11: 435-9.

13. Munchau A, Bloem BR, ThiL K.V, TRImbie MR, ROTHWEL JC, RoBERTSON M. Repetitive transcranial magnetic stimulation for Tourette syndrome. Neurology 2002; 59: 1789-91.

14. LeCKMAn J, Cohen D. Evolving Models of Pathogenesis. En: Leckman J, Cohen D eds. Tourette Syndrome: Tics, Obsessions, Compulsions. New York. Wiley \& Sons 1999; 155-76.

15. Peterson B, Leckman J, Arnsten A, Andarson G, Staib L, GoRE J ET AL Neuroanatomical Circuitry. En: Leckman J, Cohen D eds. Tourette Syndrome: Tics, obsessions, Compulsions. New York. Wiley \& Sons 1999: 230-60. 